\title{
Winners of the EFSUMB Young Investigator Award 2016
}

\section{Patient-Specific Mechanical Charac- terization Of Abdominal Aortic Aneurysms Using $4 \mathrm{D}$ Ultrasound}

E.M.J. van Disseldorp ${ }^{1,2}$, N.J. Petterson ${ }^{1}$, M.C.M. Rutten ${ }^{1}$, F.N. van de Vosse ${ }^{1}$, M.R.H. M. van Sambeek ${ }^{2}$, R.G.P. Lopata ${ }^{1}$

1. Department of Biomedical Engineering, Eindhoven University of Technology, Eindhoven, the Netherlands

2. Department of Vascular Surgery, Catharina Hospital Eindhoven, Eindhoven, the Netherlands

\section{ABSTRACT}

\section{Introduction}

Abdominal aortic aneurysms (AAA) are silent killers and the $13^{\text {th }}$ cause of death in Western society. In this study, methods for wall stress analysis (WSA) and elastography (EL) were developed using 4D ultrasound (US) to determine patient-specific wall stresses and material properties. These techniques were introduced in the clinic and tested in a subgroup of patients in an ongoing study with 300 patients in follow-up.

\section{Methods}

In forty patients (AAA diameter 27 $52 \mathrm{~mm}$ ), 4D-US data were measured using a Philips iU22 (X6 - 1 transducer). The brachial blood pressure was measured using an arm cuff. The US data were manually segmented. The patient-specific geometry was tracked over time to estimate its displacement field using $3 \mathrm{D}$ speckle tracking. Subsequently the diastolic geometry was converted into a finite element model. WSA was performed assuming a neo-Hookean material model. The model was optimized by iteratively adapting the material properties until the model output matched the $3 \mathrm{D}$ displacements. For seven patients, computed tomography (CT) data were available and used to compare the USbased geometries and wall stresses.

\section{Results}

The 4D-US based $99^{\text {th }}$ percentile wall stress ranged between 198 to $390 \mathrm{kPa}$, and the patient-specific material property $\left(G_{\text {inc }}\right)$ had a median of $1.1 \mathrm{MPa}$ (IQR: 0.7 $1.4 \mathrm{MPa}$ ). Geometry based on US data showed good similarity indices (0.90 0.96 ) with $C T$, and the $25^{\text {th }}$ to $95^{\text {th }}$ percentile wall stresses were in good agreement. Small aneurysms revealed stresses similar to those in large AAAs. Furthermore, the arterial stiffness increased with respect to AAA diameter.

\section{Conclusion}

This study shows that $4 \mathrm{D}$ US-based WSA and EL of AAAs is feasible and has the potential to aid in AAA rupture risk assessment by identifying patients at risk, and to monitor patients over time by detecting changes in wall stress and material properties. Ongoing work includes a novel automatic segmentation and registration algorithm and long-term follow-up.

Emiel M.J. van Disseldorp received his M.Sc. degree in Medical Engineering from the Eindhoven University of Technology (Eindhoven, the Netherlands) in 2014. During his Masters, he was an Intern at the University of Canterbury, Christchurch, New Zealand where he worked on a mathematical model for neurovascular coupling, which resulted in one co-authored publication. During his master thesis he worked on novel ultrasound methods to perform elastographic measurements in aneurysms in the thoracic ascending aorta in collaboration with the University Hospital of Brussels, Belgium.

He soon afterwards started his PhD, entitled "Quantitative monitoring of abdominal aortic aneurysms using 3D ultrasound" at the PULS/ e group of the Eindhoven University of Technology, and the department of Vascular Surgery of the Catharina Hospital Eindhoven. In this project, a method was developed to perform wall stress analysis and mechanical characterization of abdominal aortic aneurysms using 4D ultrasound data, which is currently validated in a large group of patients. In his relatively short career, Emiel has (co-)authored five publications and has 
won the young investigator award at the annual conferences of both the European Society of Biomechanics and the EFSUMB, both in 2016.

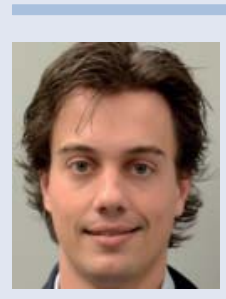

Best technical presentation - Emiel MJ van Disseldorp

\section{In vitro quantification of tissue elasticity using three shear wave elastography platforms on liver fibrosis phantoms}

Anesa Mulabecirovic , MD ${ }^{1,2}$ Anders Batman Mjelle, MD ${ }^{1,2}$ Odd Helge Gilja, MD, PhD ${ }^{1,2}$ Roald Flesland Havre, MD, PhD ${ }^{1}$

1. National Centre for Ultrasound in Gastroenterology, Department of Medicine, Haukeland University Hospital, Bergen, Norway

\section{Department of Clinical Medicine, University of Bergen, Bergen Norway}

\section{ABSTRACT}

\section{Introduction}

To assess and validate the reproducibility of quantitative elastography measurements, using shear-wave methods on four individual tissue-mimicking liver fibrosis phantoms with known Young's modulus.

\section{Methods}

We used three different shear wave elastography platforms: GE Logiq E9 SWE, Philips iu22 XM ARFI and Samsung RS80A. Both linear (high frequency) and curvilinear (low-frequency) probes were applied. The objects were four individual tissue mimicking liver fibrosis phantoms with different Young's modulus within the range of soft biological tissue $(2.7 \mathrm{kPa}, 11.5 \mathrm{kPa}, 24.8 \mathrm{kPa}$, $46.3 \mathrm{kPa}$ ). Two individual investigators performed all measurements in parallel. Each investigator made ten non-continued measurements of each phantom. The platforms were evaluated for inter- and intraobserver variability, coefficient of variation, ICC and Bland-Altman using the median value. Statistical analysis was performed with SPSS.

\section{Results}

All three elastography platforms showed excellent intra-and interobserver agreement (interclass correlation $0.981-1.000$ and intraclass correlation $0.987-1.000$ ). All four liver fibrosis phantoms could be differentiated by quantitative elastography, by all platforms $(p<0.001)$. In the BlandAltman analysis the differences in measure- ments were larger for the phantoms with higher Young's modulus. All platforms had a coefficient of variation in the range $0.00-0.21$ for all four phantoms, equivalent to low variance and high reproducibility.

\section{Conclusion}

All systems used in this study provided a high reproducibility in quantitative measurements in a liver fibrosis phantom and excellent inter- and intraclass correlations. The GE Logiq E9 SWE had the best interand intraclass correlation, whilst Philips iu22XM ARFI and Samsung R08A provided elasticity measurements closest to the elasticity values provided by the manufacturer of the phantom.

Anesa Mulabecirovic studied medicine at the Univeristy of Lubeck, Germany having spent a practical year in Bergen, Norway and was schooled in the USA. She is now working at the University of Bergen Hospital in her main interest which is elastography. Apart from English, German and Norwegian Anesa also speaks Bosnian, Serbian and Croatian.

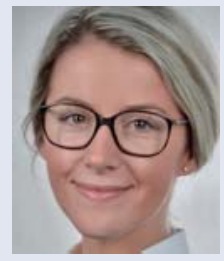

Best clinical presentation Anesa Mulabecirovic 